Recepción: 07 / 06 / 2016

Aceptación: 27 / 10 / 2016

Publicación: 06 / 01 / 2017

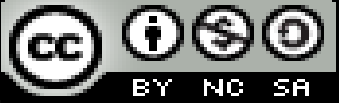

Ciencias de la salud

Artículo de investigación

\title{
Manifestaciones clínicas, psicológicas y de la sexualidad en un grupo de mujeres en el climaterio y la menopausia
}

Clinical, psychological and sexuality manifestations in a group of women in climacteric and menopause

Clínicas, manifestações psicológicas e sexualidade em um grupo de mulheres na menopausa e menopausa

Luís A. Jimbo Yepez ${ }^{\mathrm{I}}$ dr.luisy@hotmail.com

Marcelino R. Carrasco Guaman " rafaelcarrasco2688@gmail.com

Julio C. Jimbo Mendoza ${ }^{m}$ juliojimbom@hotmail.com

Correspondencia:dr.luisy@ hotmail.com

IMédico, Instituto Ecuatoriano de Seguridad Social (IESS); Guayaquil, Ecuador.

${ }^{\text {II }}$ Médico, Instituto Ecuatoriano de Seguridad Social (IESS); Guayaquil, Ecuador.

III Magister en Gerencia de Salud para el Desarrollo Local, Especialista en Gerencia y Planificación Estratégica en Salud, Doctor en Odontología, Diplomado Superior en Odontología Integral, Diploma Superior en Desarrollo Local y Salud, Docente de La Universidad Laica Eloy Alfaro de Manabí., Manta, Ecuador. 


\title{
Resumen
}

Se realizó un estudio descriptivo transversal en mujeres comprendidas entre 40 y 59 años, procedentes de la ciudad de Portoviejo, de la provincia de Manabí, Ecuador, que asistieron a consulta privada de Climaterio en el periodo de enero-julio 2016, con vista a identificar algunos aspectos biológicos, sociales y clínicos relacionados con las características de su climaterio y menopausia. Se analizaron las siguientes variables: síntomas climatéricos, esfera psicológica y con relación a la sexualidad. La unidad de resumen utilizada fue el porcentaje. Se concluyó que tanto en peri menopáusicas como en postmenopáusicas predominaron los bochornos o calores $96.3 \%$ y $99.2 \%$ respectivamente, los síntomas psíquicos están presentes en ambos grupos, los de mayor representación fueron: la ansiedad (91.6\%), el mal carácter (86.8\%), depresión y la disminución de la capacidad de tomar decisiones con (85.2\% y 85.5\%), con respecto síntomas sexuales predominó la disminución en el deseo sexual en el grupo de mujeres peri menopáusica (78.1\%), y la insatisfacción en el grupo de mujeres postmenopáusicas (74.2\%).

Palabras clave: climaterio; menopausia; manifestaciones biológicas; clínicos y sociales.

\begin{abstract}
A descriptive cross-sectional study was carried out on women between the ages of 40 and 59 from the city of Portoviejo, who attended to a Climaterium private consultation in January-July 2016, with the aim of identifying some biological, social and clinical aspects related to the characteristics of its climacteric and menopause. The following variables were analyzed: climacteric symptoms, psychological sphere and in relation to sexuality. The summary unit used was the percentage. It was concluded that in both menopausal and postmenopausal women, hot flashes predominated (96.3\% and 99.2\%, respectively). Psychological symptoms were present in both groups, with the highest number of participants being anxiety (91.6\%), in a bad mood (86.8 $\%$ ), depression and decreased ability to make decisions $(85.2 \%$ and $85.5 \%)$, with respect to sexual symptoms, decreased sexual desire in the peri-menopausal women group (78.1\%), and dissatisfaction in the group of women postmenopausal women (74.2\%).
\end{abstract}

Keywords: climacteric; menopause; biological manifestations; clinical and social. 


\section{Resumo}

Um estudo descritivo transversal em mulheres entre 40 e 59 foi realizado, a partir da cidade de Portoviejo, que participou do climatério prática privada no período de janeiro a julho de 2016, com vista a identificar alguns aspectos biológicos, sociais e clínicos relacionada com as características do seu climatério e menopausa. sintomas climatéricos e psicológica esfera em relação à sexualidade: As seguintes variáveis foram analisadas. A unidade de resumo utilizada foi a percentagem. Concluiu-se que tanto a perimenopausa e pós-menopausa predominou ondas de calor ou afrontamentos $96.3 \%$ e $99.2 \%$, respectivamente, sintomas psicológicos estão presentes em ambos os grupos os mais representados foram: ansiedade (91.6\%), mau humor (86.8\%), depressão e diminuição da capacidade de tomar decisões (85.2\% e 85.5\%) em relação a sintomas sexuais predominou diminuição do desejo sexual na perimenopausa grupo de mulheres $(78.1 \%)$ e insatisfação no grupo mulheres pós-menopáusicas (74.2\%).

Palavras chave: climatério; menopausa; manifestações biológicas; clínica e social.

\section{Introducción}

El envejecimiento, es un hecho natural que comienza con el nacimiento y en el que influyen los principios y fundamentos con los que la mujer ha vivido y lo que debería ser una etapa natural, entre la etapa reproductiva y la segunda mitad de la vida, se ha transformado en una situación muchas veces difícil de asumir, tratando de considerar el climaterio y la menopausia como una enfermedad a la que se le atribuye el fin de la sexualidad de las mujeres; ya que ésta se relaciona solamente con la capacidad para tener hijos, situación que denota que ésta etapa está rodeada de mitos miedos y temores. En la actualidad la expectativa de vida de las mujeres ha aumentado de tal manera que viven 30 años más en una etapa vital post reproductiva, situación que amerita un abordaje integral de la sexualidad, que reconozca la importancia de la función sexual como elemento que proporciona calidad de vida a las mujeres. ${ }^{1}$

El climaterio, constituye un paso evolutivo en el que la mujer transita por turbulentas experiencias que generan intensas emociones. El modo en que vive el climaterio, está predeterminado por el conjunto de valores que le fueron transmitidos verbalmente, y los que adquirió en base a cómo vivieron el climaterio las mujeres pertenecientes a los grupos en que creció y se desarrolló. ${ }^{2}$ 
A lo largo de la vida sexual de la mujer, se pueden señalar etapas que para algunas se convierten en eventos de vital importancia: la menarquía, la primera relación sexual, el embarazo, el parto y el climaterio. Diferentes estudios realizados han demostrado, que las mujeres viven la menopausia según sus factores hereditarios, dieta, estilo de vida, medio social y actitudes culturales. ${ }^{3}$

La menopausia, es un punto de inflexión para muchas mujeres debido a que marca el fin de su etapa reproductiva, sin embargo no el de su sexualidad, ésta influye en su calidad de vida y, por ello, es un constituyente importante en su diario vivir y en su salud. ${ }^{4}$

En las últimas décadas, se ha incrementado el interés por analizar cómo viven las mujeres la sexualidad durante el climaterio, debido en gran parte, a la información obtenida de los estudios realizados en la población femenina, que concluyen que existe una prevalencia de disfunción sexual de entre el $25 \%$ y $43 \%$ en mujeres de mediana edad. ${ }^{4}$

Actualmente se ha planteado que los cambios físicos, psicológicos y sociales que pueden aparecer en este periodo son los responsables de las posibles alteraciones en la función sexual. ${ }^{5}$

En el marco de las observaciones anteriores, los cambios que en la vida femenina entrañan la etapa del climaterio y la menopausia, aunque son graduales, la mayoría de las veces, no dejan de ser importantes, de ahí su innegable impacto. Tienen una profunda incidencia sobre la psiquis femenina, así como también en las manifestaciones que suelen presentarse en ellas. La mujer los percibe y repercuten sobre su imagen y autoestima, entre otras esferas. Para mantener su equilibrio psicológico ha de estar preparada emocional y racionalmente. Es una situación nueva que tiene que afrontar, no exenta de interrogantes e inseguridades. Ella experimentará toda una serie de cambios físicos y psicológicos que tendrá que aceptar e incorporar a su vida en esta nueva etapa con las menores dificultades posibles. ${ }^{6}$

La sintomatología varía mucho de una mujer a otra. Los factores psicosociales y culturales tienen una importancia considerable en la percepción por parte de las mujeres durante este período. ${ }^{6}$

Teniendo en cuenta todos estos aspectos señalados con anterioridad y considerando su importancia, hemos decidido determinar las principales manifestaciones clínicas, psicológicas y 
de la sexualidad en un grupo de mujeres en la etapa del climaterio y la menopausia, atendidas en consulta.

\section{Metodología}

Se realizó un estudio descriptivo transversal a mujeres comprendidas entre los 40 y 59 años, procedentes de la ciudad de Portoviejo, provincia de Manabí, Ecuador, que asistieron a una consulta privada de Climaterio en el periodo de enero-julio del 2016, con vista a identificar algunos aspectos biológicos, sociales y clínicos relacionados con las características de su climaterio y menopausia.

A las mujeres comprendidas en ese grupo de edad se les aplicaron los siguientes criterios de exclusión:

- Menopausia artificial.

- Tratamientos hormonales previos.

- Trabajadoras con menos de 5 años de vínculo laboral.

- Mujeres con enfermedades graves, crónicas descompensadas y enfermedades del tiroides.

La población estudio estuvo conformada por 250 mujeres, las cuales se dividieron en dos grupos las peri menopáusicas, que fueron aquellas mujeres que no habían tenido aún la menopausia para un total de 55 y las postmenopáusicas, que fueron aquellas con la menopausia ya establecida, este grupo fue de 195 mujeres.

Se analizaron las siguientes variables:

Con respecto a los síntomas climatéricos: bochornos, sudoraciones, calambres, palpitaciones, dolor de cabeza, dolores musculares y articulares, incremento de peso, trastornos del sueño, sequedad vaginal, caída del vello axilar y púbico, ardor a la micción, incontinencia urinaria.

Con respecto a la esfera psicológica: ansiedad, depresión, sentimientos de minusvalía, disminución de la capacidad de tomar decisiones, apatía, disforia, disminución de la memoria.

Con relación a la sexualidad: presencia o no de pareja estable, satisfacción, disminución del deseo sexual, disminución de la comunicación con la pareja, dispareunia. 
Se utilizaron las historias clínicas del hospital de las mujeres que participaron en el estudio, para conocer la relación nominal de mujeres con edades entre 40 y 59 años e ir descartando aquellas que tuvieron algún criterio de exclusión.

A estas mujeres se les aplicó una encuesta diseñada para cumplir con los objetivos propuestos.

\section{Resultados}

La sintomatología clínica en mujeres peri menopáusicas y posmenopáusicas se muestra en la tabla 1 en ambos grupos predominaron los bochornos o calores en un 99.2\%, en el grupo de peri menopáusicas con $93.3 \%$ y en las postmenopáusicas un $100.0 \%$, así como las sudoraciones en ambas (96.3\% y el 92.3\%). Los dolores musculares y articulares tuvieron una mayor incidencia en las posmenopáusicas; igualmente la caída vello axilar y pubiano con $92.3 \%$

Tabla 1. Distribución de las mujeres encuestadas según los síntomas clínicos y la etapa del climaterio

\begin{tabular}{|c|c|c|c|c|c|c|}
\hline \multirow{2}{*}{$\begin{array}{l}\text { Síntomas } \\
\text { Clínicos }\end{array}$} & \multicolumn{2}{|c|}{ Peri menopáusicas } & \multicolumn{2}{|c|}{ Postmenopáusicas } & \multicolumn{2}{|c|}{ Total } \\
\hline & No. & $\%$ & No. & $\%$ & No. & $\%$ \\
\hline \multicolumn{7}{|l|}{ Bochornos o } \\
\hline calores & 53 & 96.3 & 195 & 100 & 248 & 99.2 \\
\hline Sudoraciones & 53 & 96.3 & 180 & 92.3 & 233 & 93.2 \\
\hline \multicolumn{7}{|l|}{ Calambres en } \\
\hline manos y pies & 27 & 49.0 & 100 & 51.2 & 127 & 50.8 \\
\hline Palpitaciones & 48 & 87.2 & 150 & 76.9 & 198 & 79.2 \\
\hline Dolor de cabeza & 43 & 61,5 & 105 & 76.9 & 148 & 59.2 \\
\hline \multicolumn{7}{|l|}{ Dolores } \\
\hline musculares & 51 & 78.1 & 150 & 76.9 & 201 & 80.4 \\
\hline
\end{tabular}




\begin{tabular}{|l|c|c|c|c|c|c|}
\hline $\begin{array}{l}\text { Dolores } \\
\text { articulares }\end{array}$ & 25 & 45.5 & 180 & 92.3 & 205 & 82.0 \\
\hline Incremento de & 33 & 54.5 & 150 & 76.9 & 183 & 73.2 \\
\hline peso & & & & & & \\
\hline Alteraciones del & & & & & & \\
sueño & 52 & 94.4 & 90 & 46.1 & 142 & 56.8 \\
\hline Sequedad vaginal & 30 & 54.5 & 135 & 69.2 & 165 & 66.0 \\
\hline Caída vello axilar & & & & & & \\
\hline y pubiano & 13 & 23.6 & 180 & 92.3 & 193 & 77.2 \\
\hline
\end{tabular}

Como se observa en la tabla 2, muchos de los síntomas psíquicos están presentes en ambos grupos, los de mayor representación fueron: la ansiedad (91.6\%), el mal carácter (86.8\%), depresión y la disminución capacidad toma de decisiones con (85.2\% y 85.5\%). El sentimiento de minusvalía y la disminución de memoria mostraron los más bajos porcentajes.

Tabla 2. Distribución de las mujeres encuestadas según los síntomas de la esfera psicológica.

\begin{tabular}{|l|c|c|c|c|c|c|}
\hline \multirow{2}{*}{ Esfera Psicológica } & \multicolumn{2}{|c|}{ Peri menopáusicas } & Postmenopáusicas & \multicolumn{2}{|c|}{ Total } \\
\cline { 2 - 7 } & No. & $\%$ & No. & $\%$ & No. & $\%$ \\
\hline Ansiedad & 49 & 89.0 & 180 & 92.3 & 229 & 91.6 \\
\hline Depresión & 53 & 96.3 & 160 & 82.0 & 213 & 85.2 \\
\hline Sentimientos de & & & & & & \\
minusvalía & 15 & 27.2 & 60 & 30.7 & 75 & 30 \\
\hline Disminución capacidad & 38 & 69.0 & 165 & 84.6 & 213 & 85.5 \\
\hline
\end{tabular}




\begin{tabular}{|l|c|c|c|c|c|c|}
\hline toma de decisiones & \multicolumn{2}{|l|}{} & & & & \\
\hline Irritabilidad & 46 & 83.6 & 120 & 61.5 & 166 & 66.4 \\
\hline Apatía & 50 & 90.9 & 120 & 61.5 & 170 & 68.0 \\
\hline Mal carácter & 52 & 94.5 & 165 & 84.6 & 217 & 86.8 \\
\hline Disminución de la & & & & & & \\
memoria & 7 & 12.7 & 80 & 41.0 & 87 & 34.8 \\
\hline
\end{tabular}

De las 250 mujeres encuestadas, 230 refirieron tener relaciones sexuales. Como puede observarse en la tabla 3, en esta predominó la disminución del deseo sexual en el grupo de mujeres peri menopáusica (78.1\%), también estuvo presente en las postmenopáusicas para el 40.8\%; la insatisfacción sexual en las postmenopáusicas fue de $74.2 \%$ a diferencia de las peri menopáusicas.

Tabla 3. Distribución de las mujeres encuestadas según el comportamiento de los síntomas sexuales.

\begin{tabular}{|l|c|c|c|c|c|c|}
\hline \multirow{2}{*}{ Esfera Sexual } & \multicolumn{3}{|c|}{ Peri menopáusicas } & \multicolumn{2}{c|}{ Postmenopáusicas } & \multicolumn{2}{c|}{ Total } \\
\cline { 2 - 8 } & No. & $\%$ & No. & $\%$ & No. & $\%$ \\
\hline Insatisfacción & 15 & 27.2 & 130 & 74.2 & 145 & 63.0 \\
\hline Disminución del & & & & & & \\
deseo sexual & 43 & 78.1 & 94 & 53.7 & 94 & 40.8 \\
\hline Disminución de & 30 & 54.5 & 38 & 21.7 & 68 & 29.5 \\
\hline
\end{tabular}


comunicación con la

pareja

\begin{tabular}{l|l|l|l|l|l|l|}
\hline Dispareunia & 15 & 27.2 & 94 & 53.7 & 109 & 47.3 \\
\hline
\end{tabular}

\section{Discusión}

En medicina, son importantes los signos y los síntomas para llegar a un diagnóstico de lo que sucede en el organismo, y desarrollar un plan o ruta crítica de estudios para confirmarlo, que permita la certeza del tratamiento adecuado. Dependerán entonces de la etapa del climaterio en que se encuentre la mujer, además de los signos y síntomas que manifieste. Una vez que los síntomas se presentan, lo que habitualmente sucede en la segunda década del climaterio, llamado "climaterio perimenopáusico", se inicia la etapa temprana de la sintomatología. Muchas mujeres (hasta el $80 \%$ de acuerdo con estadísticas estadounidenses y entre el 40 y $76 \%$ de acuerdo con estadísticas mexicanas) experimentan "bochornos", uno de los síntomas más característicos de que se está perdiendo o se ha perdido la producción hormonal en los ovarios. Estos bochornos, o un equivalente como pueden ser episodios de sudoración nocturnos, pueden aparecer aún antes de que desaparezca la menstruación, especialmente los días previos a que llegue esta. ${ }^{7,8}$

Tanto los bochornos como los episodios de sudoración, tienen como origen un desajuste en el funcionamiento de las estructuras del sistema nervioso, encargadas de regular el control de la temperatura y los vasos sanguíneos, por lo que se han llamado cambios vasomotores. ${ }^{3}$ Los resultados del presente estudio coinciden con los de Heredia Hernández B ${ }^{6}$, quienes refirieron el mayor porciento de sintomatología en peri menopáusicas como en postmenopáusicas predominaron los calambres de manos y pies $79.5 \%$ y $82.4 \%$ respectivamente, así como los bochornos, $53.8 \%$ en peri menopáusicas y $76.5 \%$ en postmenopáusicas. La ansiedad y la depresión fueron las manifestaciones psíquicas más frecuentes; y en la esfera sexual predominó la disminución del deseo sexual en las peri menopáusicas (25.6\%) y la insatisfacción en las postmenopáusicas $(58.3 \%)$.

Si bien es cierto que ninguna mujer se muere de bochornos, estos alteran significativamente la calidad de vida de quien los padece, y si tiene vida de pareja, también su compañero y hasta su 
familia se ven afectados, ya que pueden alterar la calidad del sueño al presentarse a cualquier hora del día o de la noche, ocasionando incomodidad y despertar frecuentes. ${ }^{3}$

El comportamiento de los síntomas clínicos durante el climaterio de las pacientes estudiadas, coincide con lo descrito por la mayoría de los autores, que plantean que los síntomas vasomotores son los más frecuentes y pueden observarse entre un $75 \%$ y un $85 \%$ de las mujeres de estas edades, síntomas que tienden a disminuir después de la menopausia a pesar de persistir el hipoestrinismo. En el período posmenopáusico se intensifican los cambios genitourinarios, sobre todo la sequedad vaginal, la caída del vello axilar y púbico y la incontinencia urinaria, que a su vez pueden incrementar las disfunciones sexuales. ${ }^{9,}$ 10, 11, 6

En España, según el Dr. Rafael Sánchez-Borrego-Director de la Clínica Diatros de Barcelona y miembro de la Junta Directiva de la Asociación Española para el Estudio de la Menopausia (AEEM), se estima que más del $50 \%$ de las mujeres en etapa climatérica sufre deterioro en su calidad de vida, debido a los cambios que se producen durante la peri menopausia. Aparecen las alteraciones menstruales y se manifiestan de forma más evidente los síntomas característicos del síndrome climatérico: alteraciones neurovegetativas con síntomas vasomotores de sofocos, cambios de humor, sudación e insomnio, pueden presentarse síntomas a nivel genitourinario, como atrofia y sequedad vaginal, cambios en su sexualidad, así como pueden aparecer algunas patologías que se deben prevenir, las más frecuentes son la osteoporosis y la enfermedad cardiovascular. $^{12}$

Las manifestaciones psicológicas también están presentes, como pudo observarse en los resultados, y las mismas dependen de cómo la mujer contemple y acepte o no esta etapa de transición, como ha sido señalado. Las más frecuentes fueron la ansiedad y el mal carácter. En algunas mujeres, la peri menopausia representa un período de mayor vulnerabilidad a la aparición de síntomas depresivos. Aquellas que experimentan otros síntomas, como sofocos e insomnio, parecen encontrarse especialmente en riesgo de presentar depresión durante este período. Además, las pacientes con antecedentes de depresión, en particular durante episodios relacionados con la reproducción, también parecen tener mayor riesgo de depresión durante el período en estudio. ${ }^{13}$ 
La función sexual es la resultante de la interacción de múltiples factores en el individuo, tanto anatómicos, biológicos, fisiológicos y psicológicos y a éstos se agrega la interrelación con la pareja donde juegan un papel los patrones culturales, las vivencias previas, los sentimientos hacia la pareja, el estado marital mismo y la autoestima, mediante ella, se permite a la pareja mantener una íntima comunicación y el disfrute de sensaciones placenteras. ${ }^{1}$

Diversos estudios relacionados con la función sexual en mujeres en etapa de climaterio, encontraron que a nivel mundial se registra una prevalencia de disfunción sexual que oscila entre $20 \%$ y $30 \%$ de las mujeres en general. ${ }^{1}$

Con respecto a la sexualidad, predominó la insatisfacción en las postmenopáusicas y la disminución del deseo sexual en las peri menopáusicas. Si bien es cierto que existen cambios biológicos en esta etapa, ${ }^{14,6}$, también hay que considerar la representación que tiene lo social y lo cultural, que abarcan determinaciones que pueden repercutir desfavorablemente de acuerdo al contexto donde la mujer se desenvuelva. ${ }^{15,6}$

Según lo expuesto en una investigación realizada por Pugo Plaza J ${ }^{16}$ la cual tuvo como objetivo, determinar el grado de conocimiento que tienen las mujeres sobre el climaterio y cuál es su actitud de respuesta frente a los cambios que desencadena esta nueva etapa de vida, valorando los principales síntomas percibidos por las mujeres. En Cuenca, Ecuador, se evidenció que un 15.7\% tienen conocimiento sobre el climaterio, un $47 \%$ de mujeres encuestadas, tienen una actitud positiva frente al climaterio y el $17.3 \%$ manifiestan una actitud negativa refiriendo temor a envejecer. El principal síntoma presentando es aumento de peso con un $49.5 \%$, seguido de disminución de la libido con el 40.5\% (150) de encuestadas. Otro estudio ${ }^{17}$ ejecutado en el Ecuador, puso de manifiesto que los síntomas encontrados con mayor frecuencia fueron las oleadas de calor $(53.3 \%)$, la sudoración (49.2\%), la pérdida de memoria (80.6\%), la depresión (67.4\%), los dolores musculares y articulares (84.0\%), la resequedad de la piel (85.5\%), la evasión a la intimidad (76.2\%) y los cambios en el deseo sexual (76.5\%). La población estudiada presentó algunas similitudes y diferencias, en comparación con las mujeres menopáusicas de otros grupos étnicos.

Se concluyó que tanto en peri menopáusicas como en postmenopáusicas predominaron los bochornos o calores $96.3 \%$ y $99.2 \%$ respectivamente, los síntomas psíquicos están presentes en 
ambos grupos, los de mayor representación fueron: la ansiedad (91.6\%), el mal carácter (86.8\%), depresión y la disminución capacidad toma de decisiones con (85.2 y $85.5 \%$ ), con respecto a los síntomas sexuales, predominó la disminución en el deseo sexual en el grupo de mujeres peri menopáusica (78.1\%), y la insatisfacción en el grupo de mujeres postmenopáusicas (74.2\%).

\section{Referencias bibliográficas}

1- Rivera Moya D C, Bayona Abello S. Climaterio y función sexual: un abordaje integral a la sexualidad femenina 2014. Revista CES Salud Pública 5 (1).

2- Marchisio S, Campo C, Yuli M. Un estudio sobre el climaterio femenino a través de un cuestionario autoadministrado. Revista científica 2011 [citado 15 Ene 2016]; Disponible en: http://www.redalyc.org/html/184/18424417010/

3- Capote Bueno María Isabel, Segredo Pérez Alina María, Gómez Zayas Omar. Climaterio y menopausia. Rev Cubana Med Gen Integr. 2011 [citado 29 de enero 2016]; 27(4): 543-

557. Disponible en: http://scielo.sld.cu/scielo.php?script=sci_arttext\&pid=S0864$21252011000400013 \& \operatorname{lng}=\mathrm{es}$.

4- Sánchez Izquierdo Melodie, Hernández García Emilio. Climaterio y sexualidad. Enferm. glob. 2015 Oct [citado 27 Ene 2016]; 14 (40): 76-85. Disponible en: http://scielo.isciii.es/scielo.php?script=sci_arttext\&pid=S1695-

$61412015000400004 \& \operatorname{lng}=\mathrm{es}$.

5- Yanes Calderón M, Chio Naranjo I. Climaterio y sexualidad: su repercusión en la calidad de vida de la mujer de edad mediana. Rev Cubana Med Gen Integr. 2008 (citado 17 Dic 2015); 24(2). Disponible en:http://scielo.sld.cu/scielo.php?script=sciarttext\&pid=S086421252008000200005 .

6- Heredia Hernández Braulio, Lugones Botell Miguel. Principales manifestaciones clínicas, psicológicas y de la sexualidad en un grupo de mujeres en el climaterio y la menopausia. Rev Cubana Obstet Ginecol. 2007 [citado 27 Ene 2016]; 33(3): Disponible en: http://scielo.sld.cu/scielo.php?script=sci_arttext\&pid=S0138$600 \times 2007000300009 \& \operatorname{lng}=\mathrm{es}$. 
7- Artiles Visbal L, Manzano Ovies BR, Navarro Despaigne D. Cuerpo, sexualidad y climaterio: La necesidad de un manejo médico social integral. Rev Centroamericana Obstet Ginecol. 2002; 11(4):2-7.

8- Rojas Cabana AS. Prácticas de autocuidado que realizan mujeres premenopáusicas del Asentamiento Humano Cerro del Sauce Alto. Lima. Perú. 2003. [Citado: 11 de Enero del 2016]. Disponible en: http://www.cybertesis.edu.pe/sisbib/2003/rojas_ca/pdf/rojas_ca.pdf

9- Goldstein I, Alexander JL. Practical aspects in the management of vaginal atrophy and sexual dysfunction in perimenopausal and postmenopausal women. J Sex Med. 2005; 2(Suppl 3):154-65.

10- Álvarez Sintes R, et al. Temas de Medicina General Integral. Tomo II. La Habana: Editorial Ciencias Médicas; 2001.

11- NIH State-of-the-Science Conference Statement on management of menopause-related symptoms. NIH ConsensStateSci Statements.2005; 22(1):1-38.

12- Navarro Despaigne D. Calidad del hueso en mujeres de edad mediana. Rev. Cubana Endocrinol. 2007; 18(1). [Citado 28 de enero de 2016]. Disponible en: http://bvs.sld.cu/revistas/end/vol18_1_07/end02107.htm

13- Gyllstrom ME, Schreiner PJ, Harlow BL. Perimenopause and Depression: Strength of Association, Causal Mechanisms and Treatment Recommendations. Best Practice \& Research Clinical Obstetrics \& Gynaecology.2007; 21(2):275-92.

14- Lugones Botell M. La salud de la mujer en el climaterio y la menopausia. La Habana: Ed.Científico Técnica;2006:34

15- Sarduy Nápoles M, Lugones Botell M. II Consenso Cubano sobre Climaterio y menopausia. La Habana: Editorial CIMEQ; 2007: 12-13.

16- Pugo Plaza J. Quintuña Bravo R. Conocimientos, actitudes y sintomatología sobre el climaterio en mujeres de 45 a 65 años que acuden a consulta externa de Ginecología del Hospital Vicente Corral Moscoso (hvcm). Cuenca. 2015. 2016. 
17- Frecuencia de los síntomas de menopausia y riesgos asociados en Ecuador. Rev Panam Salud Pública. 2007 [citado 29 de Enero 2016]; 21(5): 331-332. Disponible en: http://www.scielosp.org/scielo.php?script=sci_arttext\&pid=S102049892007000400013\&lng=en. http://dx.doi.org/10.1590/S1020-49892007000400013. 